\title{
A Proposed Method for Correcting Aperture Penetration in High Energy Slit Aperture and Pinhole SPECT
}

\author{
M.C. Wrobel ${ }^{1}$, N.H. Clinthorne ${ }^{2}$, Member, IEEE, J.A. Fessler ${ }^{2}$, Member, IEEE, Y. Zhang ${ }^{2}$, S.J. Wilderman ${ }^{3}$ \\ and W.L. Rogers ${ }^{2}$, Member, IEEE \\ ${ }^{1}$ AL/OEBZ, $2402 \mathrm{E}$ Dr, Brooks AFB TX 78235-5114 \\ ${ }^{2} 3480$ Kresge III, Box 0552, University of Michigan, Ann Arbor, MI, 48109-0552 \\ ${ }^{3} 4271$ Cooley, University of Michigan, Ann Arbor, MI, 48109
}

\begin{abstract}
Aperture penetration in high energy pin-hole and slit aperture SPECT results in a decrease of both resolution and contrast. At $511 \mathrm{keV}$, approximately $50 \%$ of projection counts can be attributed to penetration. Correction for this effect by measuring the penetration from a "blocked" slit has been investigated using SPRINT-II. Slit apertures were blocked with small bars of depleted uranium (DU) and projections acquired to serve as an estimate of the penetration background. The block's finite size and DU's limited attenuation of 511 $\mathrm{keV}$ photons required the blocked projection data to be corrected by a spatially variant, Monte Carlo based window function. ${ }^{18} \mathrm{~F}$ line sources imaged without correction for penetration had an $8.8 \mathrm{~mm}$ FWHM and $18 \mathrm{~mm}$ FWTM due to large penetration tails. Lines reconstructed with penetration correction achieved $5.0 \mathrm{~mm}$ FWHM and $8.6 \mathrm{~mm}$ FWTM resolution. An ${ }^{18} \mathrm{~F}$ filled $\mu$-Jaszczak phantom (hot lines from $1.5 \mathrm{~mm}$ to $4 \mathrm{~mm}$ ) imaged without correction produced a barely discernible $4 \mathrm{~mm}$ line set and uniform background. Images produced with correction produced clear images of $3 \mathrm{~mm}$. holes with negligible background The findings should be generally applicable to clinical gamma camera systems.
\end{abstract}

\section{INTRODUCTION}

Considerable interest has developed in the use of high resolution pin-hole single photon emission computed tomography (PH SPECT) for small animal quantitative imaging [1] [2] [3] [4] [5] [6]. These works have principally investigated low-energy (140 keV) imaging due to the significant image degradation which results from aperture penetration by higher energy photons. This paper describes a novel method to correct for slit aperture penetration in high energy SPECT by measuring blocked aperture projections. The technique is applicable to both conventional filtered backprojection and iterative reconstruction methods.

Experiments were performed using SPRINT-II, a second generation full ring SPECT tomograph designed for small animal imaging of ${ }^{99 \mathrm{~m}} \mathrm{Tc}$ labeled compounds and tumor imaging of ${ }^{131} \mathrm{I}$ labeled compounds. The detector ring consists of 11 sodium iodide detector modules, each $14 \mathrm{~cm}$ by $15 \mathrm{~cm}$ by $12 \mathrm{~mm}$ located on a radius of $25 \mathrm{~cm}$. Collimation of the detectors is provided by the combination of a rotating multislit ring aperture and a stationary slice collimator which can have either a parallel or fan-beam configuration [7] [8]. The aperture ring consists of six slits, the slit edges are constructed of depleted uranium (DU) to maximize collimation. The aperture-detector arrangement with the slits at a radius of 6.4 $\mathrm{cm}$, affords an $8.75 \mathrm{~cm}$ field-of-view (FOV).

In reconstructing images acquired with slit aperture geometries, it is assumed that measured projection data is exclusively from gamma rays that pass through the slit. As gamma ray energy increases, the aperture edges become less effective attenuators and this assumption is wcakened. For SPRINT imaging $511 \mathrm{keV}$ photons, approximately half the measured projection data stems from photons that penetrate the hybrid lead-DU small animal aperture. The effect is shown in Figure 1, comparing the aperture penetration predicted by ray-tracing to that measured for an ${ }^{18} \mathrm{~F}$ line source centered in the SPRINT gantry. The normalized profile of a measured ${ }^{99 \mathrm{~m}} \mathrm{Tc}$ line source is also shown. We observe the system point response function is significantly broadened by penetration, resulting in a subsequent loss of image resolution and contrast.

Predicted vs Actual Slit Penetration at $511 \mathrm{keV}$ (2 mm slit)

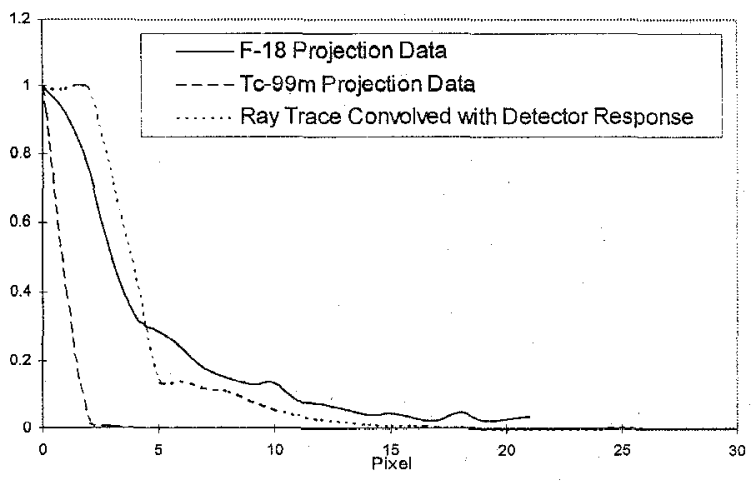

Figure 1. Demonstration of Aperture Penetration Measured for 511 $\mathrm{keV}$ Annihilation Radiation. Ray traced penetration is shown compared to measured penetration with ${ }^{18} \mathrm{~F}$.

\section{METHODS}

\section{A. Initial Attempts to Correct for Penetration}

Projection data acquired from ${ }^{18} \mathrm{~F}$ line sources indicates that the penetration component of the projection appears as a smooth background added to the true signal. This was observed to be similar in effect to scatter radiation originating from within the measured object in conventional emission tomography. Hence, methods successfully used for scatter 
correction were explored as to their efficacy in correcting aperture penetration. A technique that could be applied to existing projection data is the convolution-subtraction method originally applied to SPECT[9] and later to PET image reconstruction[10]. For application to penetration correction, the projected penetration distribution is estimated by the following analytical form:

$$
\hat{P}_{\text {Pene }}=\int_{-D}^{D} P_{\text {measured }} \cdot h(x-t, t) d t
$$

Where $h(x, t)$ is the convolution kernel describing the penetration as a function of $x$ (image plane) and $t$ (detector plane). If an accurate kernel can be determined, the true projection signal is estimated by:

$$
\hat{P}_{\text {Signal }}=P_{\text {measured }}-\int_{-D}^{D} P_{\text {measured }} \cdot h(x-t, t) d t
$$

The projection data from line sources translated across the field-of-view were measured to empirically determine the blurring (convolution) function (Figure 2). It is observed that the tails are a function of both source location within the field of view, as well as projection angle. The resulting threedimensional kernel is further complicated by being discontinuous in $\mathrm{x}$ for rays passing directly through the slit opening. Because of the complex form of the kernel, implementation of this technique was not pursued.

Similar issues discourage an iterative solution to this problem where aperture penetration is modeled and incorporated into the system response matrix. The first complication is that for each source location and for each aperture position the probability of a photon penetrating the aperture and interacting in a given detector element must be determined. This requires an extensive Monte Carlo assessment of the SPRINT imaging geometry that is both difficult and time consuming. Second, a non-sparse system matrix results from the finite probability of aperture penetration for all detector pixels from all source pixels. For SPRINT, this would require a 390 MByte weight matrix, which is too large for RAM storage on most computer platforms and substantially lengthens image reconstruction.

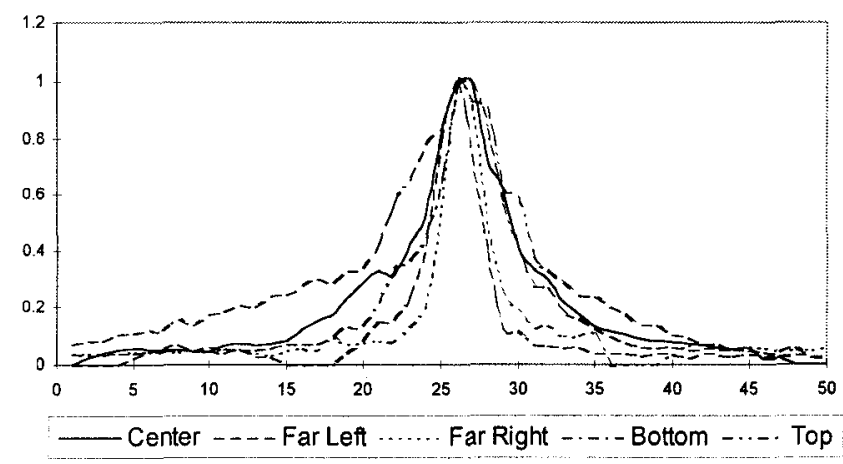

Figure 2. Spatially Variant Penetration Tails Observed for Line Sources Moved Throughout the Field-of-View. Labels represent source positions in the FOV.

\section{B. A Proposed Solution: Correction Through Blocking}

The developed method attempts to measure the aperture penetration by "blocking" the slit aperture. We observe that the measured projection data is composed of both true signal and aperture penetration (Equ. 3). If the "penetration" aspect of the projection data can somehow be measured directly or estimated from measurements, then the true projection signal is estimated from Equ 4:

$$
\begin{aligned}
& P_{\text {measured }}=P_{\text {Signal }}+P_{\text {Penetration }} \\
& \hat{P}_{\text {Signal }}=P_{\text {measured }}-\hat{P}_{\text {Penetration }} \\
& \hat{P}_{\text {Signal }}=\left(P_{\text {Signal }}+P_{\text {Penetration }}\right)-\hat{P}_{\text {Penetration }}
\end{aligned}
$$

By blocking the aperture, the measured projection data serves as an estimate of the penetration background. This can then be subtracted from the open slit projection data to obtain a true net signal. Experiments were conducted on the effect of blocking the aperture slit with a depleted uranium bar $(3.125$ $\mathrm{mm} \times 6.25 \mathrm{~mm}$ ) and measuring the resulting projection for an on-axis line source. The results are shown in Figure 3 . The blocked slit data has the expected form of the penetration signal, except that it does not drop to zero at the center of the projection. This results from the block not being completely opaque to $511 \mathrm{keV}$ gamma rays and from detector blurring. The figure also shows the net projection data resulting when the blocked slit projection data is subtracted from the open slit data. The normalized projection profile of a ${ }^{99 \mathrm{~m}} \mathrm{Tc}$ source is presented for reference. The net projection data is observed to have significantly improved resolution.

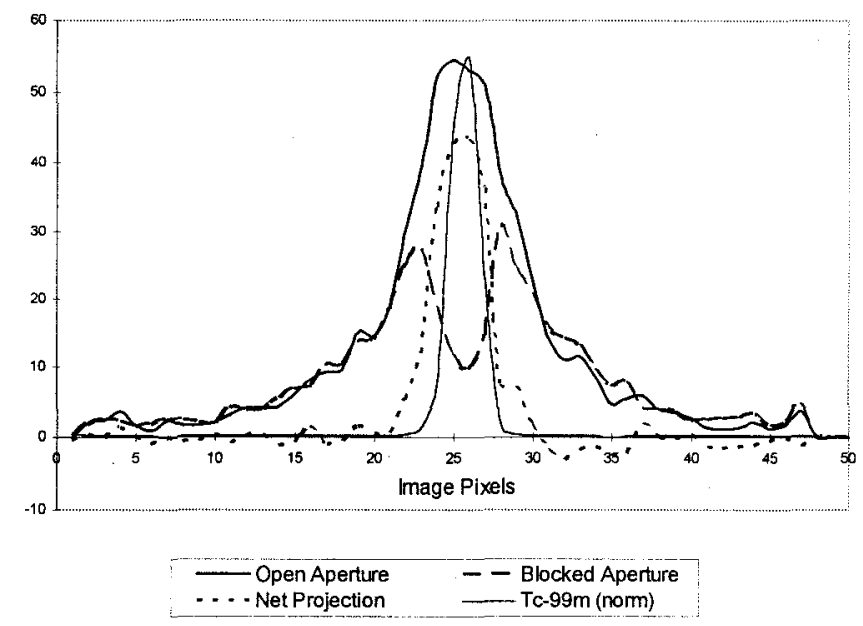

Figure 3. Projection Profiles for Open Slit and Blocked Slit Apertures

The method's efficacy was assessed for extended objects by making a series of off-axis source measurements using line sources. Figures 4 shows a consistent improvement in resolution and a reduction in the tails for the subsequent net responses. 


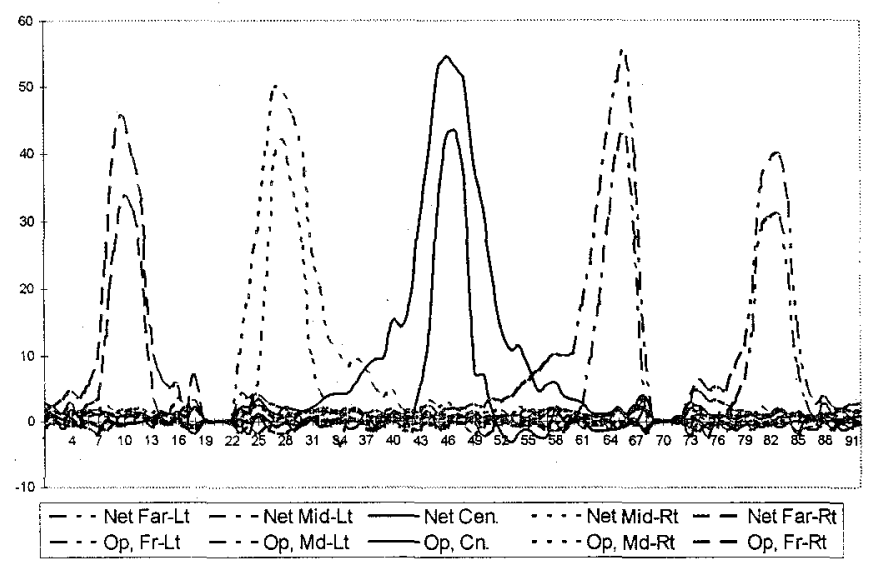

Figure 4. Open and Net Line Source Projections, the Latter Obtained from Difference Between Open and Blocked Slit Data

\section{Correcting for the Non-Ideal Block Response}

The correction described by Equ. 4 was applied to imaging a cylinder filled with a uniform distribution of activity. Reconstructed images however demonstrated a significant depression in counts in the central region of the object. Two factors contribute to this effect: the finite attenuating properties of the depleted uranium block and the finite thickness of the block. The attenuation afforded by $6.25 \mathrm{~mm}$ of DU permits approximately $10 \%$ of incident $511 \mathrm{keV}$ photons to be transmitted (based on total interaction crosssections [11]). These photons contribute to the measured blocked projections, causing an overestimate of the aperture penetration. The finite block size causes a "penumbral" effect between the aperture edge and block where photons incident on the aperture edge can also be incident on the DU bar. The result is an underestimation of the penetration data since the block introduces additional attenuation for the aperture edges.

The spatial dependence of these two effects are observed in Figure 5, where rays are traced for four source locations through the block. For points along the central axis, the block acts as a rect ( $\Pi$ ) function, attenuating the incident radiation with little penumbral effect. In contrast, off-axis locations approach a $\Lambda$ function. As off-axis distance increases, the edge interaction between the block and aperture edges becomes predominant in affecting the penetration measurement.

To evaluate a correction scheme for these effects, Monte Carlo simulations were conducted of the SPRINT aperture with $511 \mathrm{keV}$ gamma-rays [12]. The aperture was simulated using three designs: with a $3.125 \mathrm{~mm}$ open slit, with the slit blocked by the depleted uranium bar, and with the slit blocked by an infinitely thin perfect absorber. Point sources were then simulated for each of these geometries both on-axis and at four off-axis locations. To simplify implementation, the simulations did not include the detector response. Figure 6 shows the difference in detected photons incident at the detector surface between the DU blocked aperture and perfectly blocked aperture for the on-axis and two off-axis points (10\% window). For both the on-axis and off-axis cases, there is notable penetration through the bar, compared to zero flux for the perfectly blocked slit. The effect is somewhat mitigated in the off-axis cases as the bar is traversed at an angle from normal, increasing the effective thickness of the block. The penumbral effect is only demonstrated for the offaxis points. For the ideally blocked slit, there are highly peaked responses on either side of the block, associated with rays passing through region where the aperture knife edge meets the block. These responses are greatly diminished as rays incident in this area impinge on both the aperture edge and finite size DU bar.

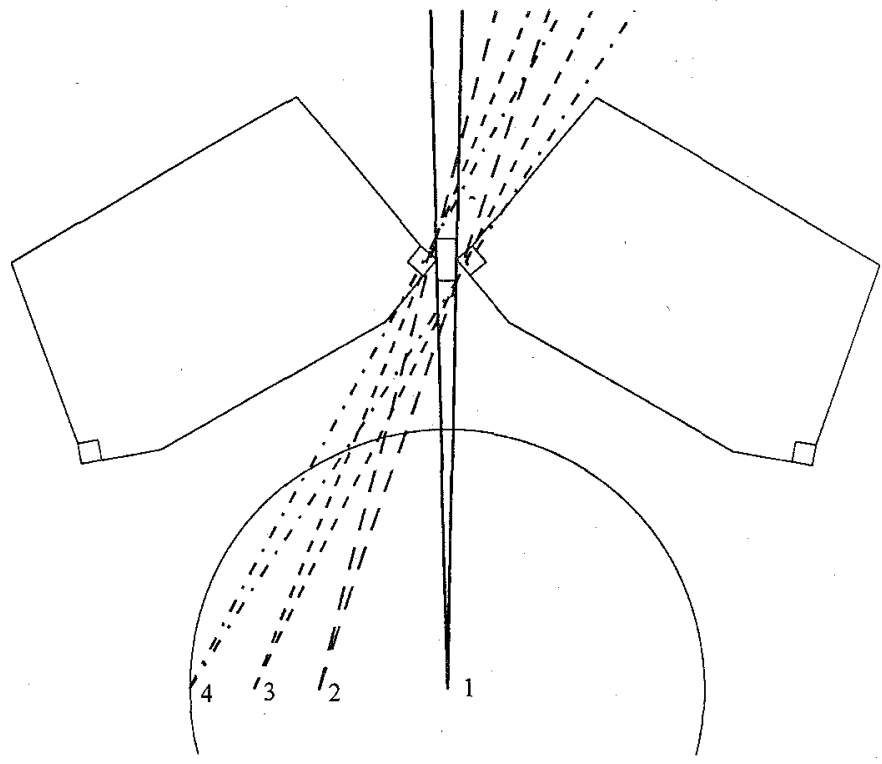

Figure 5. Ray-Tracing from Point Source Locations in Field of View Through DU Block and Aperture Edges

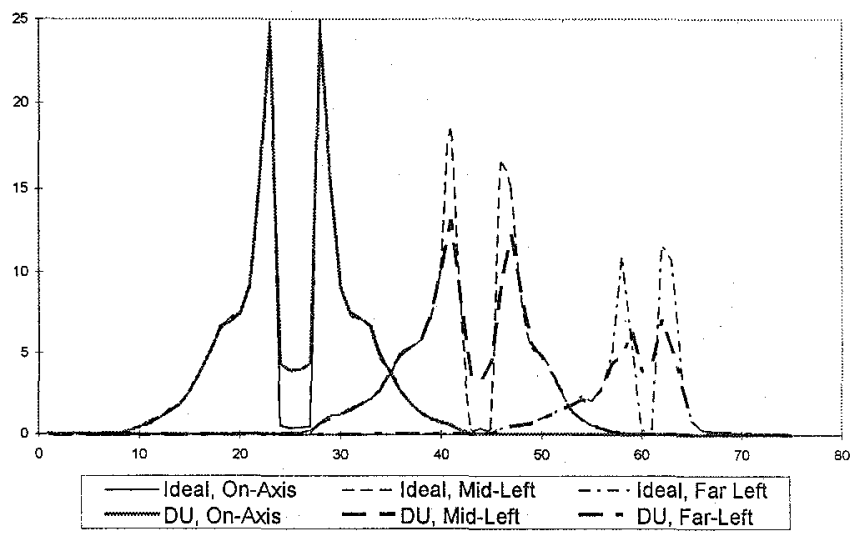

Figure 6. Comparative Point Source Profiles of Aperture Blocked with DU Bar and a Perfect Attenuator. Point sources located along line at center of field of view.

The combined effects of block penetration and penumbra are better observed in Figure 7 which shows the net difference between the DU blocked and perfectly blocked aperture. The net responses are shown for the on-axis source (solid line), followed by the four source positions out to the far left of the field of view. Values greater than zero are attributed to penetration through the aperture block, causing an overestimation in the measured penetration data. Negative 
values correspond to penumbral effects between the aperture edge and aperture block and result in an underestimation of the measured penetration.

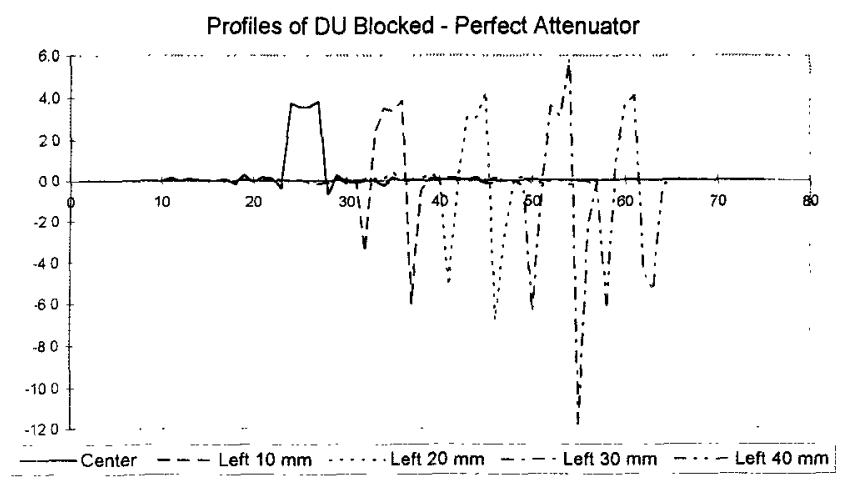

Figure 7. Simulated Assessment of Penumbral Effects and Aperture Block Penetration.

A weighting scheme for the measured block slit projection data was derived by examining the ratio of integrals of the DU blocked slit data and the perfectly blocked slit data. By plotting these normalized ratios as a function of projection centroid, the points in Figure 8 result. For the on-axis case, the ratio is approximately 1.1 , in good agreement with the expected $10 \%$ transmission through the block. As source position is moved to the far left of the field of view, the ratio approaches 0.91 , indicating the joint effects of penumbra and penetration result in off-axis positions being underestimated by $9 \%$. The Monte Carlo data were fit to a three-parameter Blackman-Gaussian function which provided a $99.7 \% \mathrm{R}^{2}$ correlation with the data (dashed line). For comparison, the figure shows an empirically derived Blackman window which was initially used to correct the projection data (solid line).

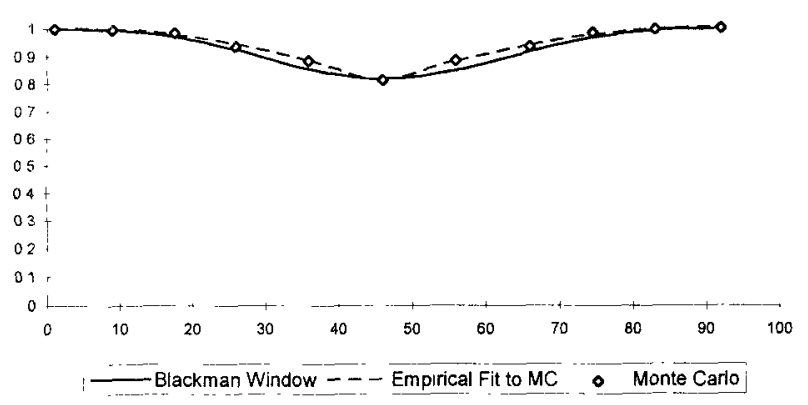

Figure 8. Comparison of Monte Carlo Based Weights to Empirically Derived Blackman Window

The simulation based function was refined by convolving a Gaussian detector response ( 2 pixel $\sigma$ ) to account for the detector blurring introduced into the simulation results. The weights were applied directly to the measured projection data as a post acquisition-processing operation.

Results of this correction are observed in Figure 9. The reconstruction of the uniform object using the uncorrected penetration data shows a central depression (Figure 9A). Filtered back-projection reconstruction with the weighted penetration data (Figure 9B) demonstrates recovery of the central area to produce a uniform field. By comparison, the image without penetration correction (Figure 9C) has approximately twice the counts of the corrected image, and blurred edges. This is in good agreement with measured projection data indicating penetration accounts for approximately $50 \%$ of the measured count rate.
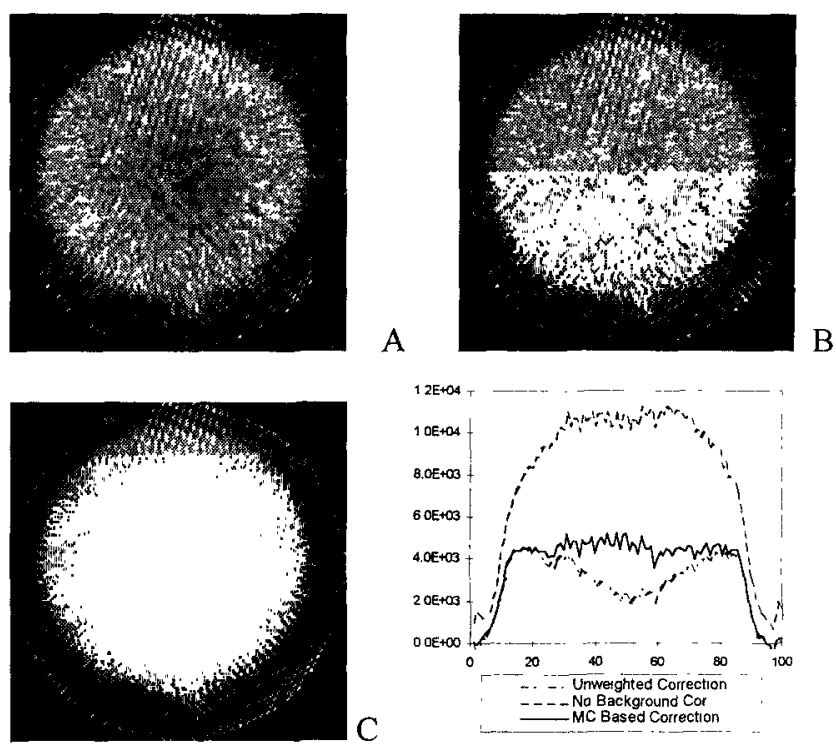

Figure 9. Effect of Imperfect Aperture Blocking. Figure A (bottom profile) show effect of directly subtracting measured penetration from open slit data. Figure B is reconstructed with weighted penetration data, and Figure $\mathrm{C}$ is reconstructed without pentration correction.

\section{Data Acquisition and Image Reconstruction}

To measure aperture penetration as part of a normal tomographic acquisition, the SPRINT slit aperture was modified so that every other slit was blocked by a depleted uranium bar. Available material dictated the bar size of 3.125 $\mathrm{mm}$ by $6.25 \mathrm{~mm}$, and $150 \mathrm{~mm}$ long. The open slit width was set equal to the bar width. A tomographic acquisition sequence was then implemented which rotated the aperture 80 steps, 1.5 degree per step, sweeping the aperture in a $120^{\circ}$ arc (Figure 10). This doubled the number of projections acquired as compared to conventional low-energy imaging, and provided an open slit and blocked slit projection for each aperture position. Post acquisition-processing of the acquired raw projection data formed two complete data sets of open and blocked slit data.
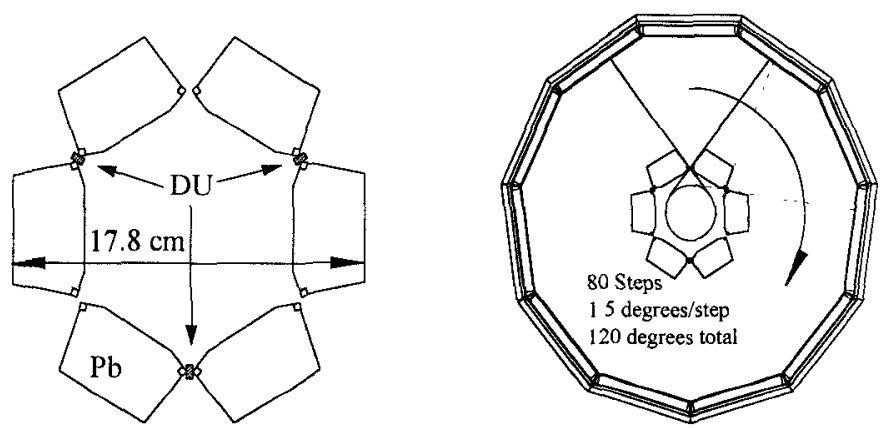

Figure 10. Slit Aperture Arrangement and Sampling Protocol for High Energy Imaging. The slit edges are composed of DU. The aperture ring rotates while the detector ring is stationary. 
Reconstruction of images was conducted using both filtered back-projection and the SAGE iterative algorithm [13]. In applying FBP, the open-slit projection data was preprocessed by subtracting the weighted blocked-slit projection data and then applying uniformity correction, $\mathrm{P}_{\mathrm{e}}$.

$$
Y_{P}=P_{e}^{-1} \cdot\left\{Y_{\text {Open }}-w Y_{\text {Blocked }}\right\}
$$

Here "w" represents the Monte Carlo derived window. High frequency noise in the reconstructed image was smoothed slightly using a Butterworth filter with a $0.9 \mathrm{~cm}^{-1}$ cutoff.

For iterative reconstruction, the SAGE algorithm was applied to compute the maximum liklihood estimate $\hat{\lambda}$ of the actual object $\lambda$, by maximizing an objective function of the form:

$$
\Phi(\lambda)=L(Y ; \lambda)-P(\lambda)
$$

where $L(Y ; \lambda)$ is the log-liklihood of the measurements $Y$, given a hypothetical image $\lambda$, and $P(x)$ is a quadratic smoothness penalty of the form:

$$
P(\lambda)=\beta \frac{1}{2} \sum_{k} \sum_{j \in N_{k}} \frac{1}{2} \omega_{k j}\left(\lambda_{k}-\lambda_{j}\right)^{2}
$$

where $\mathrm{N}_{\mathrm{k}}$ is the neighborhood of 8 pixels surrounding the kth pixel, $\omega_{k j}=1$ for horizontal and vertical neighbors and $1 / \sqrt{ } 2$ for diagonal neighbors and $\beta$ is a user specified constant.

The system model employed can be represented as:

$$
Y_{\text {Open }} \sim \operatorname{Poisson}\left\{P_{e}\left(A_{T c} \lambda+w Y_{\text {Blocked }}\right)\right\}
$$

where $A_{t c}$. are system weights generated using the geometric slit opening, and the weighted blocked slit projection data $w Y_{\text {Blocked }}$ is used as the random background term. Note that $A_{\text {tc }}$. does not include any aperture penetration effects. Regularization was performed to optimize image quality, typically in the range of $\beta=2^{-23}$ for low count images to $2^{-28}$ for high count images [13].

\section{RESULTS}

The efficacy of penetration correction was assessed using
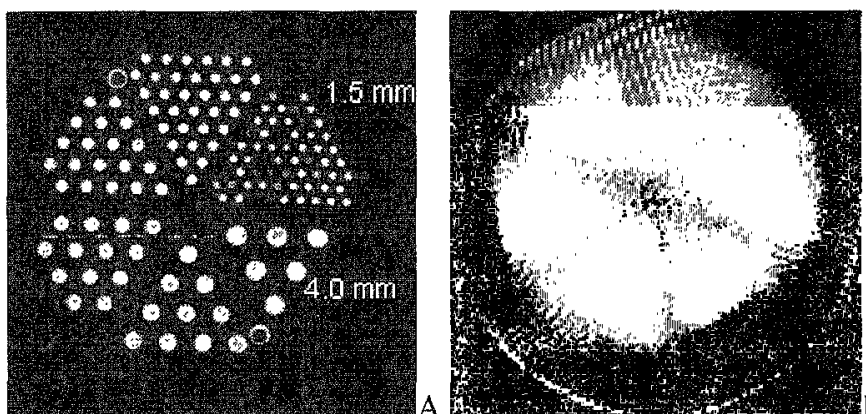

${ }^{18} \mathrm{~F}$ line sources arrayed across the field-of-view. Reconstruction performed using FBP without penetration correction is shown in Figure 11A, with the associated horizontal profile shown in Figure 11B. Qualitatively, the image suffers from poor resolution with long tails introduced on each line. There is also a tangential and radial dependence in resolution. The same lines reconstructed with penetration correction are seen in Figure 11C, with a matched profile in 11D. This image shows a significant improvement in resolution across the field of view, and resolution is now more isotropic. The tails of each source are notably reduced. Quantitatively, the non-penetration corrected image had a central resolution of $8.8 \mathrm{~mm}$ FWHM and $18 \mathrm{~mm}$ FWTM due to large penetration tails. Images reconstructed with penetration subtraction resulted in a $5.7 \mathrm{~mm}$ FWHM and a 10.8 FWTM resolution. If the blocked slit projection data are not spatially weighted and used directly for correction, a slightly better resolution of $5.0 \mathrm{~mm}$ FWHM and $8.6 \mathrm{~mm}$ FWTM is achieved due to over-correction. Images of lines reconstructed using SAGE $\left(\beta=2^{-28}\right)$ demonstrate significant additional improvements in image quality. Specifically, backprojection artifacts are removed and resolution approaches the calculated theoretical limit of $4.4 \mathrm{~mm}$, with a FWTM of $8.0 \mathrm{~mm}$.
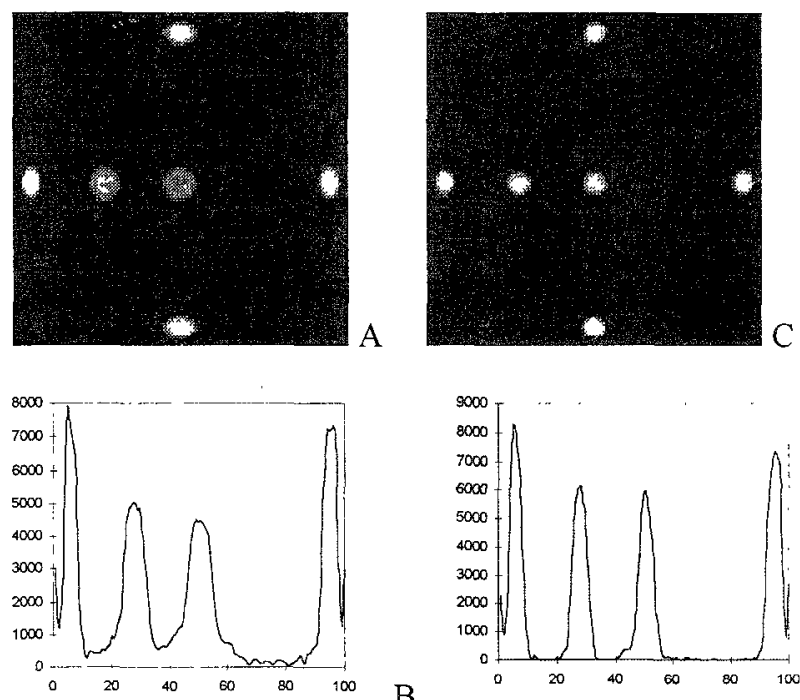

Figure 12. Six Line Source Array Reconstructed with FBP, Without and With Penetration Correction, Respectively
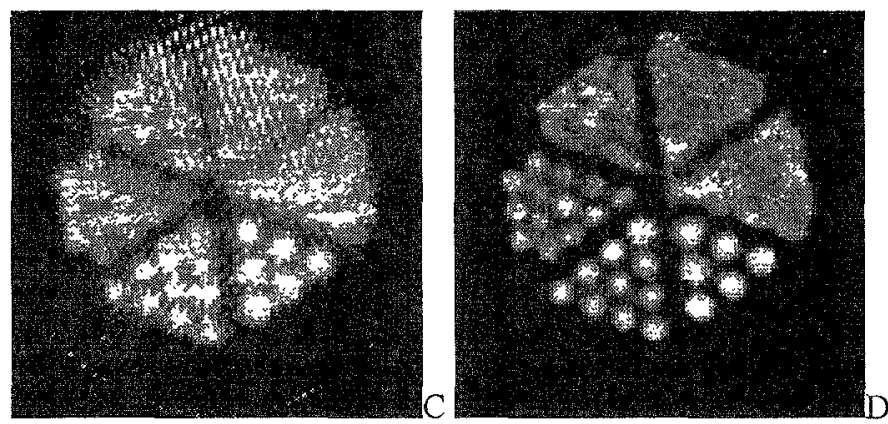

Figure 11. Images of $\mu$ Jaszczak Phantom, Hot Lines from $4.0 \mathrm{~mm}$ to $1.5 \mathrm{~mm}$. Figure A) MR image of phantom insert (rotated), B) uncorrected FBP reconstruction, C) corrected FBP reconstruction, D) corrected SAGE reconstruction. 
System sensitivity assessed after correction was $66 \pm 5.5$ $\mathrm{cpm} / \mu \mathrm{Ci}{ }^{18} \mathrm{~F}$. This very low sensitivity is attributed to the poor intrinsic scintillator efficiency $(\sim 11 \%)$, the use of a high resolution parallel slice collimator, and the additional halving of sensitivity due to this dual measurement correction method.

An ${ }^{18} \mathrm{~F}$ filled micro-Jaszczak phantom consisting of holes ranging from 1.5 to $4.0 \mathrm{~mm}$ in diameter was also imaged. Figure 12B shows a ramp filtered FBP reconstruction without penetration correction. The image was created with 87.6 million counts, yet resolution and contrast are poor. Figure $12 \mathrm{C}$ illustrates the same image reconstructed with penetration correction from 40.1 million net counts. Figure 12D demonstrates the effect of reconstructing using penetration correction and the SAGE algorithm $\left(\beta=2^{-26}\right)$. In uncorrected images, the $4 \mathrm{~mm}$ line set was barely discernible and a uniform background level was present. Images produced with correction resulted in clear observation of the $3 \mathrm{~mm}$ holes and negligible background, and the SAGE reconstruction improved resolution and reduced noise and ray artifacts.

\section{DiscUsSION}

The first order correction applied to the penetration data provides acceptable qualitative improvements in image quality. However, the weighting function, w, in Equ. 6 only approximates the effects of finite block size and attenuation for sources positioned along the diameter of the field-of-view. The effect is actually object dependent and varies over the entire slice. If the blocked projection data was biased only from penetration through the block (i.e. no penumbral effects), further improvements in image quality could be achieved through a semi iterative correction technique. The proposed system model for such a solution is:

$$
\begin{aligned}
& Y_{P} \sim \text { Poisson }\left\{P_{e}\left(A_{T c} \lambda+b\right)\right\} \\
& Y_{B} \sim \text { Poisson }\left\{P_{e}\left(\alpha A_{D U} \lambda+b\right)\right\}
\end{aligned}
$$

Where $Y_{P}$ is the measured projection data with the open aperture, $A_{T c}$ are system weights derived for the geometric slit opening, and $b$ is the background component composed of aperture penetration, scatter and environmental background. The blocked slit projection data, $Y_{B}$, is modeled as the sum of aperture penetration plus penetration (only) through the block equal to $\alpha A_{D U} \lambda$, where $A_{D U}$ are system weights generated from Monte Carlo simulations of the block penetration, and $\alpha$ is a scale factor. We estimate $b$ from the following expression, which uses the spatially weighted blocked projection data to provide a first estimate of the object, $\hat{\lambda}$ FBP-SAGE, assessed as above:

$$
\hat{b}=P_{e}^{-1}\left[Y_{B}\right]-\alpha A_{D U} \hat{\lambda}_{(F B P-S A G E)}
$$

This estimate of $b$ can then be used in equation 10(a) to solve for the object distribution using a discrete estimation algorithm, such as SAGE. Equations 10 (a) and 11 can then be solved iteratively to form improved estimates of the object distribution, $\hat{\lambda}$.
The potential for implementing this method was investigated through a series of Monte Carlo simulations of the SPRINT aperture blocked by a cylindrical DU bar. Projection data was obtained for the five point source locations used in the previous simulations. Figure 13 shows the comparison between the cylinder blocked aperture and the perfectly blocked aperture. Comparing this figure with the profiles in Figure 6, we observe that the cylinder is clearly a less effective attenuator, allowing more primary photons to be transmitted.

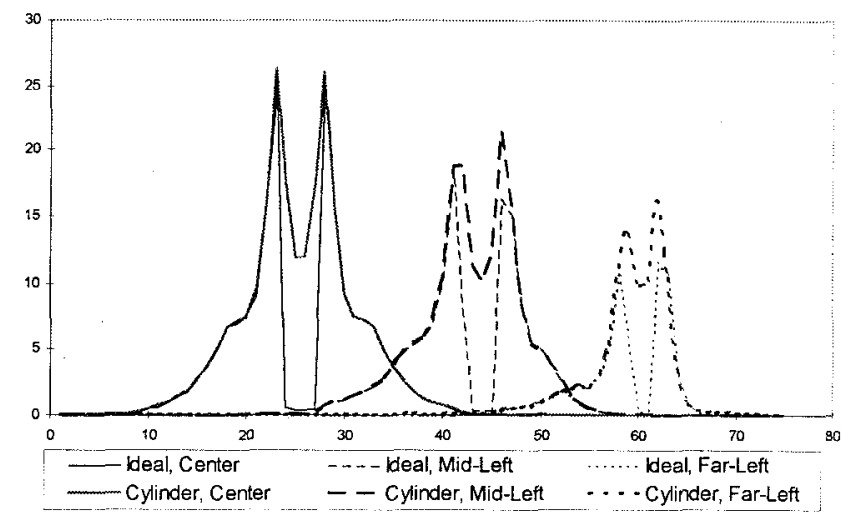

Figure 13. Comparative Point Source Profiles of Aperture Blocked with Cylindrical DU Bar and a Perfect Attenuator.

The advantage of using a cylindrical block becomes apparent when the net penetration through the cylinder is determined from the perfectly blocked aperture. This is shown in Figure 14 as the heavy lined profiles for each of the five source locations. If these profiles are compared to those shown in Figure 7, it is noted that the underestimation of the penetration data is eliminated. The resulting overestimation of the penetration data has a form which can be readily incorporated into a sparse weights matrix, $A_{D U}$. As proof, point sources at the same positions used in the simulations were forward projected using $\mathrm{A}_{\mathrm{Tc}}$ generated with a $3 \mathrm{~mm}$ slit. The resulting projections were normalized and overlaid onto the net cylindrical penetration profiles, shown as the lighter profiles in Figure 14.

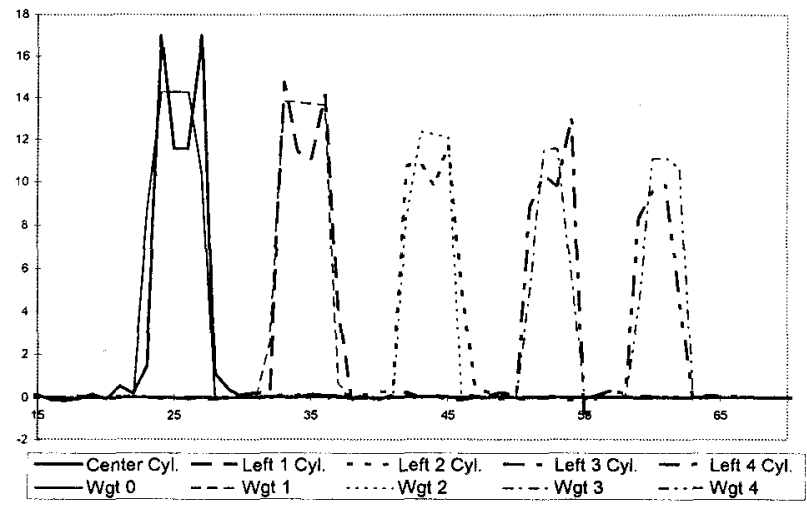

Figure 14: Comparison of the Net Penetration Through a DU Cylinder Compared to Forward Projected Point Sources Using SPRINT System Weights

We observe that the desired $A_{D U}$ is not substantially different from $\mathrm{A}_{\mathrm{Tc}}$. The good agreement between the two sets 
of data suggests that even the use of a geometric weights matrix, $\mathrm{A}_{\mathrm{Tc}}$, may provide good reconstructions using this iterative block penetration technique. Optimizing a weights matrix to match the Monte Carlo based results should provide reconstructions with improved resolution and sensitivity over the basic technique.

\section{SUMMARY}

Clear improvement in SPECT imaging of high energy gamma-rays can be obtained through the proposed penetration correction method. The method allows recovery of resolution to within one $\mathrm{mm}$ of the system geometric resolution, and significantly improves contrast by removing the long penetration tails of the system point response function. Further improvements in the blocked aperture technique are predicted using iterative reconstruction methods that accurately model the imperfect attenuation of the depleted uranium block. Such methods should provide an improvement in both sensitivity and resolution.

There are two important disadvantages of such a method, however. First, the protocol requires measuring two complete data sets to arrive at an estimate of the true signal. System sensitivity is thus effectively halved. Second, the variance in the image is increased by two times the variance of the penetration background, since this background is measured twice (reference Equ. 3). We observe that if the penetration background were modeled and included in the reconstruction process, there would be a substantial increase in sensitivity and decrease in signal variance. Unfortunately, this latter approach is complicated to implement, and would require the use of a full and potentially three-dimensional system matrix to accurately represent penetration in pin-hole geometries.

Although the effects are not as dramatic, pin-hole imaging of ${ }^{131}$ I labeled radiotracers is also significantly degraded by aperture penetration. Application of this method is expected be even more effective for $364 \mathrm{keV}$ imaging due to better attenuation characteristics of the block, and the capacity to correct for high energy iodine emissions The devised method should be germane to both planar and SPECT imaging, and be well suited to clinical application on two-head and four-head commercial gamma cameras.

\section{REFERENCES}

[1] J. Palmer and P. Wollmer. "Pinhole emission computed tomography: method and experimental evaluation" Phys Med Biol vol. 35, pp. 339-350, 1990.

[2] R.H. Moore, H. Ohtani, B.A. Khaw and H.W. Strauss. "High resolution pin-hole sequence imaging of small laboratory animals". J Nucl Biol vol. 32, pp.987, 1991.

[3] D.A. Weber, M. Ivanovic, D. Franceschi, S.E. Strand, K. Erlandsson, M. Franceschi, H.L. Atkins, J.A. Coderre, H. Susskinf, T. Button et.al. "Pinhole SPECT: an approach to in-vivo high resolution SPECT imaging in small animals" J Nucl Med vol. 35(2), pp. 342-8, 1994.

[4] R.J. Jaszcak, J. Li, H. Wang, M.R. Zalutsky, and R.E. Coleman, "Pinhole collimation for ultra-high resolution small field-of-view SPECT studies," Phys Med Biol, vol. 39, pp.425-437, 1994.

[5] J. Li, R.J. Jaszcak, K.L. Greer, R.E. Coleman, "A filtered backprojection algorithm for pinhole SPECT with a displaced center or rotation," Phys Med Biol, vol. 39, pp.165-176, 1994.

[6] S.E. Strand, M. Ivanovic, K. Erlandsson, D. Franceschi, T. Button, K. Sjogren, D.A. Weber, "Small animal imaging with pinhole single-photon emission computed tomography," Cancer, vol. 73(3 suppl), pp.981-4, 1994.

[7] W.L. Rogers, N.H. Clinthorne, L. Shao, P. Chiao, Y. Ding, J.A. Stamos, and K.F. Koral, "SPRINT II: A Second Generation Single Photon Ring Tomograph" IEEE Trans Med Imag, vol. 7, pp.291-7, Dec 1988.

[8] M.C. Wrobel, C. Ng, N.H. Clinthorne, T.A. Webster, Y. Zhang, W.L. Rogers, "Design, construction and performace of one-dimensional lead foil parallel plate and fan-beam collimators for gamma-ray imaging", Nuc Inst Meth A, vol. 376, pp.477-483, 1996.

[9] B. Axelsson, P. Msaki, A. Israelsson. "Subtraction of Compton-scattered photons in single-photon emission computerized tomography," J Nucl Med, vol. 25, pp.490. 494, Apr 1984.

[10] M. Bergstrom, L. Eriksson, C. Bohm, G. Blomqvist, J, Litton, "Correction for scattered radiation in a ring detector positron camera by integral transformation of the projections," J Comput Assist Tomog, vol. 7(1), pp.4250, Feb. 1983.

[11] National Nuclear Data Center, Brookhaven National Laboratory, Upton NY 11973.

[12] S.J. Wilderman, "Vectorized algorithms for Monte Carlo simulation of kilovolt electron and photon transport" University of Michigan, School of Engineering, Department of Nuclear Engineering, Dissertation Thesis, 1990.

[13] J.A. Fessler and A.O. Hero, "Space-alternating generalized expectation-maximization algorithm." IEEE Transactions on Signal Processing," vol. 42(10):2664$2677,1994$. 